\title{
Effects of Different Physical Characteristics on the Compression Molding Quality of Dried Fish Floss
}

\author{
Hongmei Xu, Li Zong, and Shengfa Yuan \\ College of Engineering and Technology, Huazhong Agricultural University, \\ Wuhan 430070, China \\ xhm790912@163.com
}

\begin{abstract}
The compression molding of dried fish floss is of great significance to the development of fish product and the storage and transport of fish. In this issue, the compression ratio, relaxation ratio and shatter resistance are selected as the evaluation indicators, effects of particle size, moisture content, amount, and processing methods (whether cooked or not) on the compression molding quality of dried fish floss are analyzed. The results showed that: 1) the moisture content and amount of dried fish floss have a significant impact on the thickness of molding block, and the lower the moisture content is, the larger the thickness of molding block is, the thickness of molding block decreases with the amount of dried fish floss; 2) processing methods have great effect on the relaxation ratio of molding block, the relaxation ratio of molding block will increase if the dried fish floss is cooked;3) the amount and moisture content affect the shatter resistance of molding block observably, the greater the amount and moisture content of dried fish floss ,the better the shatter resistance of molding block is when the amount and moisture content respectively varies in the range of $3 \mathrm{~g}-5 \mathrm{~g}$ and $5.13 \%-23.57 \%$.
\end{abstract}

Keywords: Dried Fish Floss, Compression Molding, Relaxation Ratio, Shatter Resistance, Moisture Content, Particle Size.

\section{Introduction}

Fish food is not only delicious and tasty, but also rich in nutrients. Many nutrients, such as protein, vitamins, various mineral elements necessary for human body, are derived from the fish food. As it turns out, fish food has always been popular with people. Dried fish floss is a kind of fish product, which is made with delicate techniques such as cooking, meat picking, seasoning, squeezing, frying etc.The compression molding is to suppress the loose dried fish floss under external force. As a result, the volume of dried fish floss decreases, while the density increases. Nowadays, the process of compression molding has been widely used in different fields. The experimental study of food and biomass forming has become a research focus. Regarding the relationship between the physical characteristics of materials, compression process parameters and the relaxation ratio and durability of molding block, many research results and conclusions have been achieved[1-5]. However, the research on compression molding mainly focus on the processing of agricultural materials, the 
compression molding of meat product is less involved. The compression molding of meat product is lack of reference.

\section{Materials and Methods}

\subsection{Materials}

For this experiment, the salted and dried chub was broken into fish floss by a highspeed meatball machine.

\subsection{Devices}

(1) RGT2000-10 Microcomputer Control Electronic Universal Testing Machine Maximum load: 5KN; Test speed: 0.01-500mm/min; Manufacturer: Shenzhen Reger instrument Co., Ltd.

(2) YXQ.SG41.280 Portable Pressure Steam Sterilizer

Maximum working pressure: $0.15 \mathrm{MPa}$; Maximum working temperature: $126^{\circ} \mathrm{C}$; Net weight: $15.5 \mathrm{~kg}$.

(3) 202-00 Desktop Electric Oven

Temperature fluctuation: $0.1{ }^{\circ} \mathrm{C}$; Temperature range: $50-250^{\circ} \mathrm{C}$; Manufacturer: Tianjin Taisite instrument Co., Ltd.

(4) HSN-22 high-speed meatball machine

Productivity: $80 \mathrm{~kg} / \mathrm{h}$; Power: $2.2 \mathrm{kw}$.

(5) MP200-1 electronic scale

Range: 200g; Accuracy: 0.01g.

(6) Mold

A set of steel cylinder mold (Figure1) with a pressure device was designed for the experiment. The internal diameter of the mold is $30 \mathrm{~mm}$. As shown in figure 1 , the part 2 and 3 respectively denote the punch and die of the mold.

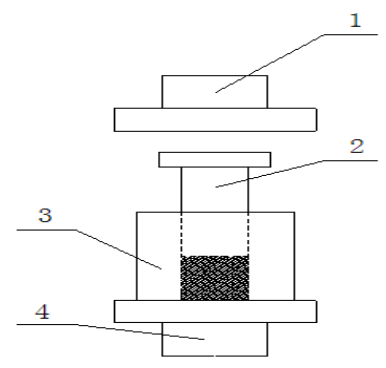

Fig. 1. Sketch map of the mold

1. Upper platen 2. Punch 3. Die 4. Bottom platen 


\subsection{Methods}

The compression molding test was conducted on the RGT2000-10 computercontrolled electronic universal testing machine. Different from other common testing machines, the compression speed and load of the universal testing machine can be adjusted. The compression experiment of the dried fish floss material was carried out at the room temperature. During the measurement, the die was placed on the bottom platen. The dried fish floss was first metered into the die, and then the punch was inserted in the die. Afterwards, adjust the location of the upper and bottom platen, so that the upper platen just contact with the punch.

Since the testing machine can't control the compression load precisely, the experiment accomplishes the indirect control of compression load by controlling the compressive strength. The compressive strength remains unchanged for sixty seconds when it reaches the pre-set maximum value. Extract the molding block, and measure the thickness of molding block within ten seconds. In order to reduce the measurement error, the thickness of molding block was measured continuously two times in the two different directions of diameter, and the final value was the averaged one.

The objective of our study was to investigate the relaxation characteristics of dried fish floss and analyze the influence of processing methods, amount, particle size, and moisture content on the quality of molding block. Accordingly, the testing program was compiled on the principle of multi-level and single-factor.

The specific programs were described as follows:

(1) In order to investigate the effect of processing methods (whether cooked or not) on the compression molding quality of dried fish floss, the comparative tests of compression molding were carried out when the other conditions remain the same. Table 1 shows the test sequence. As shown in the table, eight molding blocks were compressed for each kind of fish floss.

(2) Other things being equal, the compression molding test was performed to study on the effects of amount on the compression molding quality of fish floss. For each amount level, three molding blocks were compressed to reduce the measurement error. Table 2 shows the test sequence.

(3) In the case of other identical conditions, the compression molding test was carried out to analyze the effects of particle size on the compression molding quality of fish floss. As a matter of convenience, the experiment accomplishes the indirect control of particle size by controlling the smashing time of dried fish. Table 3 shows the test sequence. As shown in the table, four molding blocks were compressed for each particle size.

(4) Other factors being equal, the compression molding test was performed to investigate the effects of moisture content on the compression molding quality of fish floss. For convenience, the experiment accomplishes the indirect control of moisture content by controlling the drying time of fish floss. Table 4 shows the test sequence of specimens with different moisture content. Three molding blocks were compressed for each kind of fish floss with specific moisture content. 


\section{Results and Discussion}

\subsection{Relaxation Characteristics of Molding Block}

Cook chub for twenty minutes, and break it into fish floss in a high-speed meatball machine. Afterwards, the weighted fish floss was compressed into molding blocks. Figure 2 shows the relaxation characteristic curve of molding block. As shown in the figure, the thickness of molding block increases with time. Thirty minutes later, there is no significant change of thickness, and the molding block is in a state of complete relaxation.

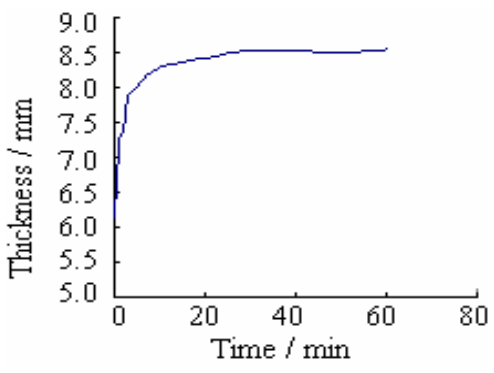

Fig. 2. Relaxation characteristic curve of molding block

\subsection{Effect of Processing Methods on the Compressibility of Fish Floss and Block Quality}

Figure 3 shows the compression curves of fish floss with different processing methods. As shown in the figure, there is a consistent variation tendency for the curves. In the beginning, a smaller compressive force can usually result in a large amount of compression. With the increase of compressive force, the displacement variation of fish floss decreased gradually. When the compressive force increases to a certain extent, the displacement variation falls to almost zero. Compare the compression curves of cooked floss with those of the uncooked, it can be found that the compression curves of cooked floss are mainly concentrated in the first half part of map, and share higher similarity with each other, whereas, those of the uncooked are loosely distributed in the second half of map. This phenomenon shows that the uncooked fish floss can obtain larger compression ratio when pressed with the identical compressive force.

Most material contracts when subjected to external forces, which usually results in the reduction of volume and increase of density. The contraction characteristic is known as compressibility. Compressibility is usually represented by the percentage of volume reduction (compression ratio) or bulk modulus.

In the study, the compressibility of fish floss is expressed as the volume compression ratio $C$, which can be calculated as follows:

$$
C=\frac{\Delta V}{V} \times 100 \%=\frac{\pi R^{2} h^{\prime}}{\pi R^{2} h} \times 100 \%=\frac{h^{\prime}}{h} \times 100 \%
$$

where $h$ ' refers to the displacement of upper platen during the process of compression molding, $h$ denotes the thickness of fish floss in the die before compression. 


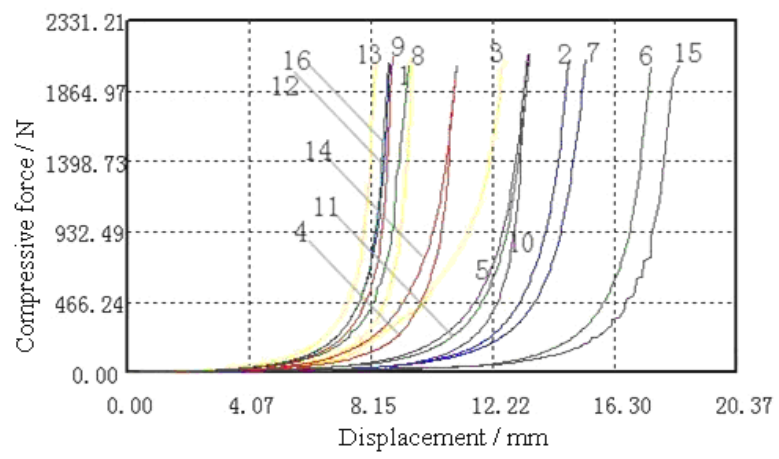

Fig. 3. Compression curves of fish floss with different processing methods 1, 4, 5, 8, 9, 12, 13, and 16: compression curves of the cooked fish floss $2,3,6,7,10,11,14$, and 15: compression curves of the uncooked fish floss

Table 1. Test sequence of the specimens when using different processing methods

\begin{tabular}{|lllllllllllllllll|}
\hline Test number & 1 & 2 & 3 & 4 & 5 & 6 & 7 & 8 & 9 & 10 & 11 & 12 & 13 & 14 & 15 & 16 \\
\hline Method & $\mathrm{Y}$ & $\mathrm{N}$ & $\mathrm{N}$ & $\mathrm{Y}$ & $\mathrm{Y}$ & $\mathrm{N}$ & $\mathrm{N}$ & $\mathrm{Y}$ & $\mathrm{Y}$ & $\mathrm{N}$ & $\mathrm{N}$ & $\mathrm{Y}$ & $\mathrm{Y}$ & $\mathrm{N}$ & $\mathrm{N}$ & $\mathrm{Y}$ \\
\hline
\end{tabular}

Note: Y (Yes) shows "cooked"; N (No) shows "uncooked"

Table 2. Test sequence of the specimens with different amount

\begin{tabular}{|llllllllllllllll|}
\hline Test number & 1 & 2 & 3 & 4 & 5 & 6 & 7 & 8 & 9 & 10 & 11 & 12 & 13 & 14 & 15 \\
\hline Amount / g & 4 & 4 & 6 & 4 & 5 & 7 & 3 & 6 & 3 & 5 & 6 & 3 & 7 & 7 & 5 \\
\hline
\end{tabular}

Table 3. Test sequence of the specimens with different particle size

\begin{tabular}{|ccccccccccccccccc|}
\hline Test number & 1 & 2 & 3 & 4 & 5 & 6 & 7 & 8 & 9 & 10 & 11 & 12 & 13 & 14 & 15 & 16 \\
\hline $\begin{array}{c}\text { Smashing } \\
\text { time / s }\end{array}$ & 30 & 30 & 10 & 40 & 20 & 10 & 30 & 10 & 20 & 40 & 20 & 40 & 20 & 10 & 30 & 40 \\
\hline
\end{tabular}


Table 4. Test sequence of the specimens with different moisture content (drying time)

\begin{tabular}{|ccccccccccccc|}
\hline $\begin{array}{c}\text { Test } \\
\text { number }\end{array}$ & 1 & 2 & 3 & 4 & 5 & 6 & 7 & 8 & 9 & 10 & 11 & 12 \\
\hline $\begin{array}{c}\text { Drying } \\
\text { time / s }\end{array}$ & 0 & 0 & 0 & 10 & 10 & 10 & 20 & 20 & 20 & 40 & 40 & 40 \\
\hline
\end{tabular}

Table 5. Average and variance of the fish floss compression ratio when using different processing methods

\begin{tabular}{|ccccc|}
\hline Group & Point & Sum & Average & Variance \\
\hline Uncooked & 8 & 5.52 & 0.69 & 0.00 \\
Cooked & 8 & 4.89 & 0.61 & 0.00 \\
\hline
\end{tabular}

Table 5 shows the compression ratio of fish floss when employing different processing methods (whether cooked or not). It can be seen that processing methods have a great impact on the compression ratio of dried fish floss, and the uncooked fish floss can obtain better compressibility.

Table 6. Average and variance of the molding block thickness when using different processing methods

\begin{tabular}{|ccccc|}
\hline Group & Point & Sum & Average & Variance \\
\hline Uncooked & 8 & 51.6 & 6.45 & 0.02 \\
Cooked & 8 & 49.2 & 6.15 & 0.06 \\
\hline
\end{tabular}

Table 6 shows the average and variance of molding block thickness when using different processing methods. The results show that processing methods affect the thickness of molding block significantly, and the molding block of uncooked fish floss is much thicker than that of the cooked.

Table 7. Average and variance of the molding block relaxation ratio when using different processing methods

\begin{tabular}{|ccccc|}
\hline Group & Point & Sum & Average & Variance \\
\hline Uncooked & 8 & 10.1 & 1.26 & 0.00 \\
Cooked & 8 & 10.9 & 1.37 & 0.00 \\
\hline
\end{tabular}

Table 7 shows the average and variance of molding block relaxation ratio when using different processing methods. As shown in the table, processing methods have great effect on the relaxation ratio of molding block; the relaxation ratio of molding block will increase if fish floss is cooked. 
Table 8. Average and variance of the molding block shatter resistance when using different processing methods

\begin{tabular}{|ccccc|}
\hline Group & Point & Sum & Average & Variance \\
\hline Uncooked & 8 & 7.88 & 0.98 & 0.00 \\
Cooked & 8 & 6.94 & 0.99 & 0.00 \\
\hline
\end{tabular}

Table 8 shows the average and variance of molding block shatter resistance when using different processing methods. The results indicate that processing methods have no significant effect on the shatter resistance of molding block, and there is no obvious difference in the shatter resistance between the cooked and uncooked.

\subsection{Effect of Amount on the Compressibility and Block Quality of Fish Floss}

Figure 4 shows the compression curves of fish floss with different amount. As shown in the figure, the three compression curves of $3 \mathrm{~g}$ fish floss are located in the farthest left of map, followed by those of $4 \mathrm{~g}, 5 \mathrm{~g}$ and $6 \mathrm{~g}$ fish floss. The compression curves of $7 \mathrm{~g}$ fish floss are located in the farthest right. Additionally, the larger the amount of fish floss is, the longer the distance traveled by testing machine platen is. Moreover, with the increase of fish floss amount, the gradients of the compression curves decrease. It is, therefore, believed that the fish floss has taken shape when the increase of compressive force hardly gives rise to the displacement variation. That is to say, the less the fish floss amount is, the smaller the force required for taking shape is.

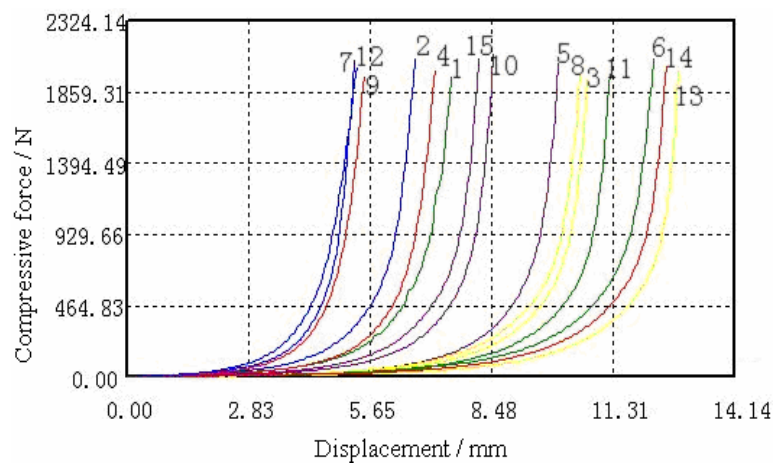

Fig. 4. Compression curves of fish floss with different amount

7, 9 and 12: compression curves of $3 \mathrm{~g}$ fish floss;

1,2 and 4 : compression curves of $4 \mathrm{~g}$ fish floss

5,10 and 15: compression curves of $5 \mathrm{~g}$ fish floss;

3,8 and 11: compression curves of $6 \mathrm{~g}$ fish floss

6,13 and 14: compression curves of $7 \mathrm{~g}$ fish floss

In order to investigate the effect of amount on the compressibility of fish floss, the compression ratio of fish floss was calculated according to Eq. (1). Table 9 shows the fish floss compression ratio with different amounts. The results of variance analysis reveal that the amount of fish floss has no significant effect on the compressibility. 
In addition, the thickness, relaxation ratio and shatter times were also calculated to analyze the effect of amount on block quality, and the results were respectively shown in Table 10, Table 11 and Table 12.

Table 9. Compression ratio of fish floss with different amounts

\begin{tabular}{|cccccc|}
\hline Amount / g & 3 & 4 & 5 & 6 & 7 \\
\hline $\begin{array}{c}\text { Compression } \\
\text { ratio \% }\end{array}$ & 59.6 & 59.6 & 58.9 & 59.0 & 58.3 \\
\hline
\end{tabular}

Table 10. Thickness of molding block with different amounts

\begin{tabular}{|cccccc|}
\hline Amount / g & 3 & 4 & 5 & 6 & 7 \\
\hline $\begin{array}{c}\text { Thickness } \\
\text { / mm }\end{array}$ & 3.69 & 4.86 & 6.23 & 7.56 & 9.01 \\
\hline
\end{tabular}

Table 11. Average and variance of the molding block relaxation ratio with different amounts

\begin{tabular}{|ccccc|}
\hline Group & Point & Sum & Average & Variance \\
\hline $3 \mathrm{~g}$ & 3 & 4.09 & 1.36 & 0.00 \\
$4 \mathrm{~g}$ & 2 & 2.79 & 1.40 & 0.00 \\
$5 \mathrm{~g}$ & 3 & 4.08 & 1.36 & 0.00 \\
$6 \mathrm{~g}$ & 3 & 4.06 & 1.35 & 0.00 \\
$7 \mathrm{~g}$ & 3 & 4.02 & 1.34 & 0.00 \\
\hline
\end{tabular}

Table 12. Average and variance of the molding block shatter times with different amounts

\begin{tabular}{|ccccc|}
\hline Group & Point & Sum & Average & Variance \\
\hline $3 \mathrm{~g}$ & 3 & 2.95 & 0.98 & 0.00 \\
$4 \mathrm{~g}$ & 2 & 1.98 & 0.99 & 0.00 \\
$5 \mathrm{~g}$ & 3 & 2.96 & 0.99 & 0.00 \\
$6 \mathrm{~g}$ & 3 & 2.98 & 0.99 & 0.00 \\
$7 \mathrm{~g}$ & 3 & 2.98 & 0.99 & 0.00 \\
\hline
\end{tabular}

On the basis of the variance analysis of experimental data, the following conclusions were derived:

1) The amount of fish floss affects the molding block thickness significantly, and the relationship between amount and thickness can be represented mathematically as follows:

$$
h=1.334 w-0.4
$$

where $\mathrm{h}$ and $\mathrm{w}$ respectively denote the molding block thickness and amount of fish floss. 
2) The amount of fish floss has no significant effect on the relaxation ratio of molding block.

3) The amount of fish floss does not significantly affect the shatter times, that is, it has no great impact on the shatter resistance of molding block.

\subsection{Effect of Particle Size on the Compressibility and Block Quality of Fish Floss}

Figure 5 displays the compression curves of fish floss with different particle size or drying time. It's clear that there are no significant differences between the curves in their variation tendency. Beyond that, these curves course irregularly. This demonstrates that in the smashing time range of $10 \mathrm{~s} \sim 40 \mathrm{~s}$, the forming speed doesn't subject to the impact of particle size. Namely, particle size has no significant effect on the compression process of fish floss.

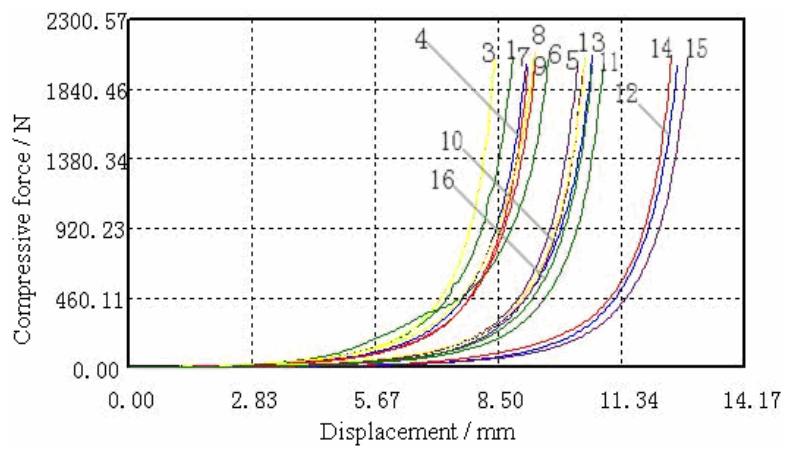

Fig. 5. Compression curves of fish floss with different particle sizes

$3,6,8$ and 14: compression curves of fish floss with the drying time of $10 \mathrm{~s}$ 5, 9, 11 and 13: compression curves of fish floss with the drying time of $20 \mathrm{~s}$

1, 2, 7 and 15: compression curves of fish floss with the drying time of $30 \mathrm{~s}$ 4, 10, 12 and 16: compression curves of fish floss with the drying time of $40 \mathrm{~s}$

Document research indicates that particle size might be a factor affecting the compressibility and block quality of fish floss. To illustrate this point, the compression ratio of fish floss, thickness, relaxation ratio, and shatter resistance of molding block were tested and calculated, and the results were respectively displayed in Table 13 16.The results suggest that particle size has no significant effect on the compression ratio of fish floss, thickness, relaxation ratio, and shatter resistance of molding block. That is to say, particle size does not affect the compressibility and block quality of fish floss significantly.

Table 13. Compression ratio of the fish floss with different particle size

\begin{tabular}{|ccccc|}
\hline Smash time/s & 10 & 20 & 30 & 40 \\
\hline $\begin{array}{c}\text { Compression } \\
\text { ratio } \%\end{array}$ & 60.46 & 61.76 & 61.89 & 62.69 \\
\hline
\end{tabular}


Table 14. Average and variance of the molding block thickness with different particle size

\begin{tabular}{|ccccc|}
\hline Group & Point & Sum & Average & Variance \\
\hline $10 \mathrm{~s}$ & 4 & 25.91 & 6.48 & 0.01 \\
$20 \mathrm{~s}$ & 4 & 25.53 & 6.38 & 0.01 \\
$30 \mathrm{~s}$ & 4 & 25.36 & 6.34 & 0.01 \\
$40 \mathrm{~s}$ & 4 & 25.47 & 6.37 & 0.01 \\
\hline
\end{tabular}

Table 15. Average and variance of the molding block relaxation ratio with different particle size

\begin{tabular}{|ccccc|}
\hline Group & Point & Sum & Average & Variance \\
\hline $10 \mathrm{~s}$ & 4 & 5.44 & 1.36 & 0.00 \\
$20 \mathrm{~s}$ & 4 & 5.56 & 1.39 & 0.00 \\
$30 \mathrm{~s}$ & 4 & 5.39 & 1.35 & 0.00 \\
$40 \mathrm{~s}$ & 4 & 5.44 & 1.36 & 0.00 \\
\hline
\end{tabular}

Table 16. Average and variance of the molding block shatter resistance with different particle size

\begin{tabular}{|ccccc|}
\hline Group & Point & Sum & Average & Variance \\
\hline $10 \mathrm{~s}$ & 4 & 3.96 & 0.99 & 0.00 \\
$20 \mathrm{~s}$ & 4 & 3.90 & 0.97 & 0.00 \\
$30 \mathrm{~s}$ & 4 & 3.96 & 0.99 & 0.00 \\
$40 \mathrm{~s}$ & 4 & 3.95 & 0.99 & 0.00 \\
\hline
\end{tabular}

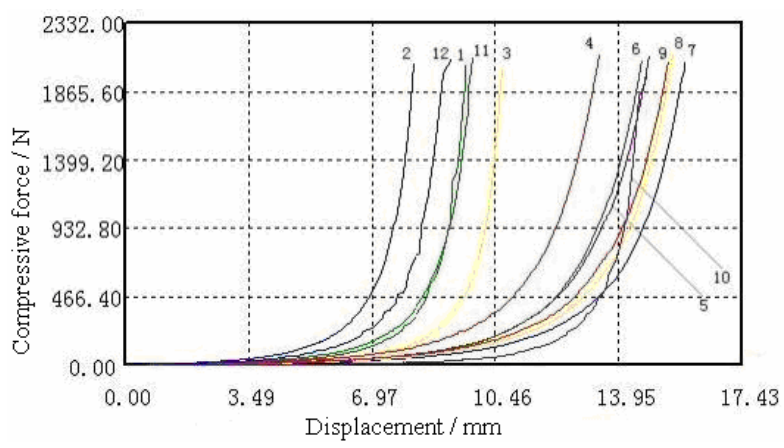

Fig. 6. Compression curves of fish floss with different moisture content

1, 2 and 3: compression curves of fish floss with the moisture content of $23.57 \%$ 4, 5 and 6: compression curves of fish floss with the moisture content of $19.32 \%$ 7, 8 and 9: compression curves of fish floss with the moisture content of $10.39 \%$ 10, 11 and 12: compression curves of fish floss with the moisture content of 5.13\% 


\subsection{Effect of Moisture Content on the Compressibility and Block Quality of Fish Floss}

Figure 6 shows the compression curves of fish floss with different moisture content. As shown in the figure, the compression curves of fish floss with the moisture content of $23.57 \%$ and $5.13 \%$ are concentrated in the first half of figure, and share great similarity with each other, whereas, those of fish floss with moisture content of $19.32 \%$ and $10.39 \%$ are mainly located in the second half part. Additionally, the gradient variation of compression curve varies with its location in the figure. The gradient variation of compression curves with moisture content of $23.57 \%$ and $5.13 \%$ is much larger than those of the others, which shows that the compressibility of fish floss varies with its moisture content.

The moisture content of fish floss mainly depends on its drying time. Table 17 shows the moisture content and compression ratio of fish floss with different drying time. It's clear that the moisture content has great impact on the compression ratio of fish floss. The correlation between the compression ratio and moisture content is presented in Fig.7.The figure reveals that compression ratio increases nonlinearly with the moisture content of fish floss, and the maximal compression ratio is achieved at the moisture content of $20 \%$.After that, the compression ratio decreases with the increase of moisture content. In other words, the moisture content is not as high as possible. In order to obtain better compressibility, the moisture content must be controlled in a certain range.

Table 17. Moisture content and compression ratio of fish floss with different drying time

\begin{tabular}{|ccccc|}
\hline Drying time/min & 0 & 10 & 20 & 40 \\
\hline Moisture content \% & 23.57 & 19.32 & 10.39 & 5.13 \\
Compression ratio\% & 61.26 & 71.54 & 66.46 & 55.80 \\
\hline
\end{tabular}

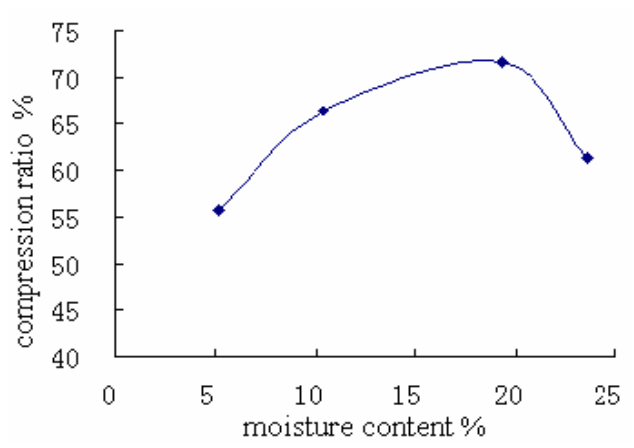

Fig. 7. Relationship between the compression ratio and moisture content of fish floss

Furthermore, in order to investigate the effect of moisture content on molding block quality, the thickness and relaxation ratio of block were also measured and calculated. Since the molding block with drying time of 40 minutes is relatively loose, it's difficult 
to analyze the variance of relaxation ratio and shatter resistance. Consequently, the tests and variance analysis don't allow for the particular case. Table 18 and Table 19 respectively display the variance analysis results of the block thickness and relaxation ratio. It can be seen that the moisture content has extremely notable effect on the thickness of molding block. Generally, the longer the drying time of fish floss, the thicker the molding block. Moreover, the drying time or moisture content has no significant effect on the relaxation ratio of molding block.

Table 18. Average and variance of the molding block thickness with different drying time

\begin{tabular}{|ccccc|}
\hline Group & Point & Sum & Average & Variance \\
\hline 0min & 3 & 17.95 & 5.98 & 0.00 \\
10min & 3 & 17.09 & 5.70 & 0.01 \\
20min & 3 & 23.65 & 7.88 & 0.02 \\
$40 \mathrm{~min}$ & 3 & 26.17 & 8.72 & 0.35 \\
\hline
\end{tabular}

Table 19. Average and variance of the molding block relaxation ratio with different drying time

\begin{tabular}{|ccccc|}
\hline Group & Point & Sum & Average & Variance \\
\hline Omin & 3 & 4.20 & 1.40 & 0.00 \\
10min & 3 & 4.06 & 1.35 & 0.00 \\
$20 \mathrm{~min}$ & 3 & 3.89 & 1.30 & 0.01 \\
\hline
\end{tabular}

\section{Conclusions}

Taking the compression ratio, relaxation ratio and shatter resistance as evaluation indicators, the effects of floss amount, processing methods, particle size and moisture content on the compressibility and block quality were discussed. The conclusions are summarized as follows:

1) Processing methods (whether cooked or not) affect the relaxation ratio significantly, and the block relaxation ratio will increase if fish floss is cooked; processing methods have no significant effect on the block thickness and shatter resistance.

2) When the floss amount varies in the range of $3 g \sim 5 g$, amount has great impact on the block thickness and shatter resistance. Generally, the greater the floss amount is, the thicker the molding block is, and the better the shatter resistance is, too. However, for the relaxation ratio, floss amount is not a significant influence factor.

3) In the smashing time range from $10 \mathrm{~s}$ to $40 \mathrm{~s}$, smashing time or particle size has no significant effect on the compression ratio of fish floss, thickness, relaxation ratio, and shatter resistance of molding block. 
4) When the moisture content varies in the range of $5.13 \%-23.57 \%$, moisture content has strong influence on the block thickness. The lower the moisture content of fish floss, the thicker the molding block. The moisture content has no significant effect on the relaxation ratio of molding block. Moreover, the moisture content affects the compression ratio of fish floss significantly. With the increase of moisture content, the compression ratio of fish floss increases firstly and then decreases. The maximal compression ratio is achieved at the moisture content of $20 \%$.

\section{Acknowledgement}

This research was supported by Scientific Research Foundation of Huazhong Agricultural University for the Introduced Talents "Research on the Evaluation Index System of Rice Moisture for Safe Storage" under grant Nos. 52204-08079.

\section{References}

[1] Hu, J.J.: Straw Pellet Fuel Cold Molding by Compression Experimental Study and Numerical Simulation.Thesis for doctor degree, Dalian University of Technology (2008)

[2] He, X.F., Lei, T.Z., Li, Z.F.: Experimental study on the cold forming technology of biomass pellet fuel. Acta Energiae Solaris Sinica 9, 937-941 (2006)

[3] Wu, J., Sheng, K.C.: Experimental Studies on Chopping Cotton Stalk When Compressed to High Densities. Journal of Shihezi University (Natural Science) 7(3), 235-238 (2003)

[4] Xing, L., Wang, S.Y., Liu, X.D.: Experiment and Analysis of Straw Compression. Journal of Jiamusi University (Natural Science Edition) 23(4), 574-576 (2005)

[5] Wang, H.B., Wang, C.G.: Study on the Stress-relaxation of Hay. Journal of Agricultural Mechanization Research 1, 134-137 (2008)

\section{Biography}

XU Hongmei received the B.S and M.S degree from Huazhong Agriculture University, and the Ph.D. degree from Zhejiang University, in 2001 ,2004, and 2008,respectively. she is currently the lecturer of the Department of Engineering and Technology at Huazhong Agricultural University. Her primary professional interests lie in digital simulation and analysis of the NVH performance for automobiles, advanced design theory and method of engine, vibration and noise control of automobile and engine, modern signal process research on vibration-noise signal, and agricultural product processing technology. 